\title{
Design and implementation of the Android client in the mobile-type teagarden environment monitoring system
}

\author{
Zhou $\mathrm{Li}^{1,}$, , Zhang Shuhui ${ }^{1, \mathrm{~b}}$ \\ ${ }^{1}$ Nanjing Institute of Industry Technology, Nanjing, 210046, China \\ aemail: seuzhouli@163.com, bemail: zsh6889@gmail.com
}

Keywords: Teagarden; Android Platform; Mobile Monitoring; Java

\begin{abstract}
An Android client of the mobile-type teagarden environment monitoring system is designed and implemented. Based on the android platform, the software programming in an Android device for monitoring the teagarden is implemented using Java language and Eclipse kit. Experiments indicate that the geographical position, data and working status of the sensors can be displayed in the mobile devices in real time. The developing approach of this new system was general-purposed. The test and application results of this system showed that this system was benefit for the agricultural production in practice with excellent features as portable, friendly interface and unlimited with networks.
\end{abstract}

\section{Introduction}

The frost damage caused by the late spring cold is very harmful to crops such as tea leaves, fruit trees and mulberry trees. It is the key factor restricting the stable development of crop yields. Domestic and foreign advanced teagarden actively introduce airflow disturbance, sprinkler [1] [2] [3] [4] to protect the crops from frost. Because the late spring cold always goes with the radiation inversion [5] [6], in the airflow perturbation method the warm air above is blown to the tea tree canopy using the fans. This method is widely recognized as an economical and effective way [7] where the most critical aspect is real time environmental monitoring in teagardens.

In recent years, with the development of smartphones in the world, it has an important role for designing one monitoring system for ordinary farmers which is more simple, friendly and portable to play realistic significance for timely and effective promotion of modern agricultural technology. The latest statistics show that the market share of Android phones is $84.6 \%$ and Android tablet is $65.8 \%$ [8]. Android operating system which is open-source and free not only provides a more flexible and independent design space for software designer, but also provides a better development platform for research and development of more humane monitoring system. A mobile-type real time environmental monitoring system based on the Android platform has been designed and implemented in this paper. This system can display the position and temperature \& humidity and sensors in the map of mobile devices in real time and can execute historical data query, data downloading and data analysis.

\section{The overall design of the system}

The mobile-type real time environmental monitoring system is composed of sensors, wireless transfer network, a server of data collection \& storage and mobile devices. Figure 1 show the overall system structure.

The core function of the system is to realize wireless transmission of the data and to display in the map in the Android devices. The main functional requirement will be showed as follows.

(1) The Server where the data collection program and web service running listens for requests.

(2) The client sends a query to the server with a username and password. If this user passes the authentication, the server sends the data of temperature \& humidity back.

(3) According to the request, the server needs to make the corresponding response. Users can specify a time period and data display style when sending out the instruction to the server. 


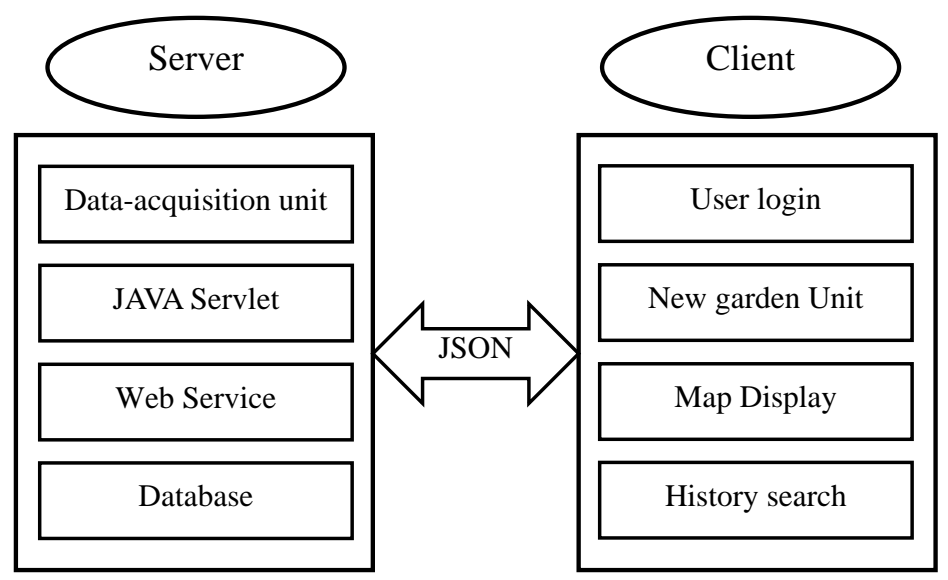

Fig.1. The overall structure of the system

(4) After parsing the request from the client, the server transfers the data back in JSON format.

(5) In order to ensure that the monitoring data is legitimate access, user authentication must be made in the server for each request from all clients.

\section{Server-side Software Development}

We accept the data from the center node of wireless sensor network using a computer connected to the node which is provided as a server for web service and data query \& analysis. A data receiving program running in the server is used to collect temperature \& humidity data and stored in the database. The data collection process will be shown in Figure 2.

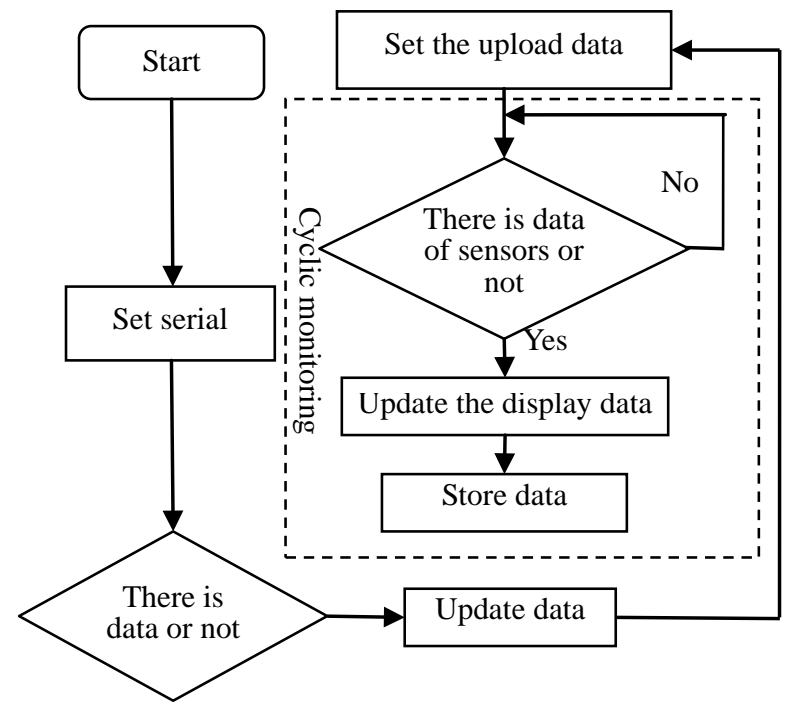

Fig.2. Data collection process

Network service program in the server has been developed with Java using DAO design pattern. DAO is a standard mode module for J2EE which can be used to build a powerful database access layer because this pattern can make developers involved in business logic layer by encapsulating the data access.

We design JSP/Servlet + EJB web applications based on MVC design mode through which users can access the data in the server anywhere on a variety of platforms. The system also realized the historical queries, data analysis and users can look up the temperature \& humidity trend map.

\section{The Client Designing and Implementation}

This paper constructs universal application systems development environment which is composed of JDK, Eclipse, Android SDK and ADT. JDK which is the core of Java includes Java runtime environment, toolkit and foundation classes. Eclipse platform is an integrated development 
environment for open source, extensible and based on Java. Android SDK provided by Google is completely open source software development kit which is specially used to develop software based on Android. ADT is a plugin for Eclipse that provides a suite of tools that are integrated with the Eclipse IDE. Figure 3 shows the system development environment.

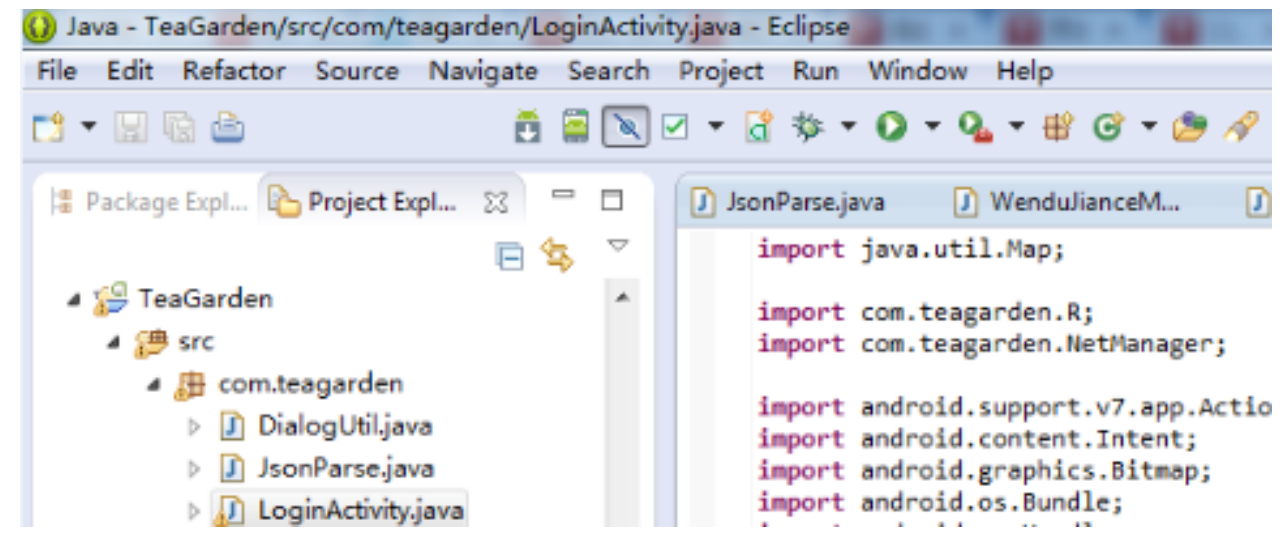

Fig.3. System development environment

The client which can manage data of one or more tea garden is composed of five parts: users login, creating tea garden, selecting garden, data display in map and historical query.

Users login unit obtains Authenticode using method httpResponse.getEntity( ) firstly and shows it in the interface. The user name and password will be sent to the server with the Authenticode. The client login authentications will succeed if the server sent 200 status codes back. Next when accessing some pages that required login permissions Cookie need to be attached to send.

Creating garden unit saves the information of garden in the SQLite3 which is integrated and embedded relational database on the Android platform. Latitude and longitude data of garden vertex can either use professional tools to measure or use the phone with a GPS device to directly access.

According users' selection, selecting unit reads latitude and longitude data of garden vertex from SQLite3 database which will be transmitted to data display unit through Intent. Data display unit shows the map in the client by means of Baidu maps SDK BaiduMap.getMap( ). The location and shape of the garden will be shown in the map by adding polygon cover. At the same time, sensors are shown in the map and the temperature \& humidity data are shown at the position of sensors. The data are accepted from the server by the way of JSON. The file "JsonParse.java" in the directory "src" realizes the function of achieving the JSON data from the server and parsing the data.

Historical query unit realized the historical queries, data analysis and users can look up the temperature \& humidity trend map.

\section{System Implementation and Application}

Using a simple, friendly man-machine interface which can show visual display monitoring information is an important prerequisite for the effective promotion of agricultural modernization technology.

This system has been installed and run in the Maichun tea garden in the city of Zhenjiang of Jiangsu province in China. All functions are demonstrated to be correct upon the test results, and reach the anticipative goal. Especially the effects of simplifying installation and wiring are notable.

The mobile real-time monitoring APP will show the map of tea garden and the position \& data of sensors directly. Our mobile device is the Samsung Galaxy Nexus phone whose android system is version 4.3. The monitoring system in this paper can be compatible with all versions of the Android system. Figure 5 show the interface of the APP. This system can manage a plurality of garden and users can choose a garden by clicking the drop-down box in the main interface. Then after clicking "OK" button the display unit show the map, sensors and temperature \& humidity data of tea garden. Users can add a sensor anytime by entering the location data in the input box above and can delete a sensor by clicking the "Delete" button appeared above it after clicking it. This system can be easily applied to a new garden which can be created by clicking the "New Garden" button. Any garden 
that has been selected will be deleted when the "Delete Garden" button is clicked. Users can know the variation and spatial distribution of temperature \& humidity whenever and wherever needed. Once data show that the phenomenon of frost happened, managers can send commands to start the fan at any time to achieve the purpose of protecting the crops from frost.

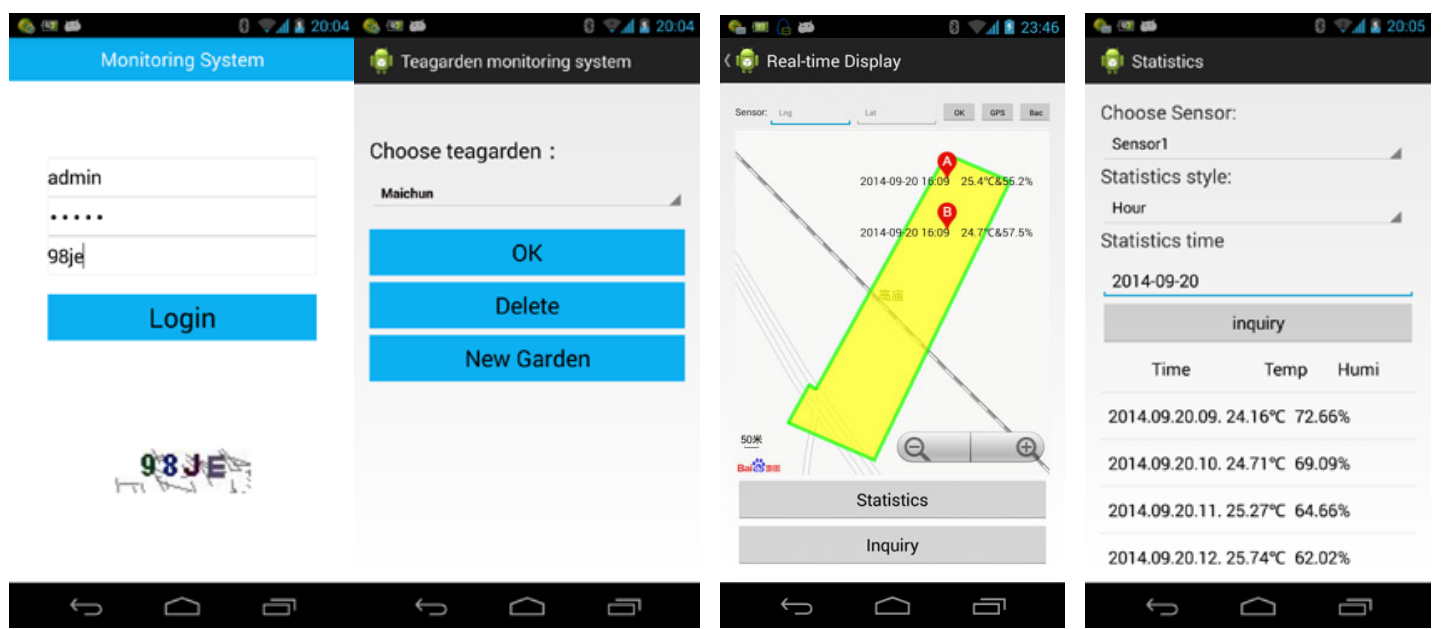

Fig.4. The mobile-type monitoring system running interface

\section{Conclusion}

In this paper, a design approach of environment monitoring system based on Android platform is described. The system designed and implemented in this paper which work over Wi-Fi, 3G and 4G wireless network has characteristics such as movability, simple installation, build up freely and communication range unlimited by environment. At the same time, the system has good flexibility, scalability and practicality. Users can add more new type sensors such as Illumination, carbon dioxide conveniently. Moreover, for new gardens this system can build the new map to monitor the new environment when adding the information of sensors. This system could be readily converted to adapt to the new environment monitoring.

\section{References}

[1] Yu Genmei. Occurrence of the Freezing Disaster of Tea Tree and its Prevention and Remedy [J], Modern Agricultural Science and Technology, 2010(10), 92 94.

[2] Xin Chongheng.: The Freezing Disaster of Tea Tree in Shandong Province and Protection Technology[J], China Tea, 2009, 31(2), 24 25.

[3] Wu Lihui. The Reason of the Freezing Disaster of Teagarden and its Protection Technology[J], Fujian Tea, 2010, 32(11), 28 29.

[4] Lu Wenyuan, Qian Wenchun, Gu Ze, etc. Frost Protection Effects of Blower System in Anji Baicha Tea Garden[J], Journal of Tea, 2009, 35(4), 215 218.

[5]Xiao Tongyu, Ren Hongyu and Zhang Xingwen. Analysis and Prediction of the Winter Inversion in Harbin[J], Journal of Nottheast Agricultural University, 2001,32(2), 105 110.

[6]KRASOVITSKI B, KIMMEL E, AMIR I. Forecasting Earth Surface Temperature for the Optimal Application of Frost Protection Methods[J]. Journal of Agricultural Engineering Research, 1996(63):93 102.

[7]Luo Yaoping. Occurrence of the Freezing Disaster of Tea Tree and its Prevention[J], China Tea, 2008, 30(1):30 31

[8]Aimo. The Android Market Share overall suppression of iOS. [EB/OL], http://pcedu.pconline.com.cn/522/5224302.html, 2014-08-06. 